\title{
Conformational Analysis of Phloroglucinols from Hypericum Brasiliense by using X-ray Diffraction and Molecular Modeling
}

\author{
Kátia Z. Leal, ${ }^{*, a, b}$ Julliane D. Yoneda, ${ }^{c}$ Eric B. Lindgren, ${ }^{a}$ Carlos B. Pinheiro, ${ }^{d}$ \\ Arthur L. Corrêa $a^{a}$ and Hildegardo S. França ${ }^{e}$ \\ ${ }^{a}$ Departamento de Físico-Química, Instituto de Química, Universidade Federal Fluminense, \\ 24020-150 Niterói-RJ, Brazil \\ ${ }^{b}$ Programa de Pós-Graduação em Química, Instituto de Química, Universidade Federal Fluminense, \\ 24020-150 Niterói-RJ, Brazil \\ 'Pólo Universitário de Volta Redonda, Universidade Federal Fluminense, \\ 27255-125 Volta Redonda-RJ, Brazil \\ ${ }^{d}$ Departamento de Física, Universidade Federal de Minas Gerais, \\ 31270-901 Belo Horizonte-MG, Brazil. \\ eDepartamento de Tecnologia Farmacêutica, Faculdade de Farmácia, Universidade Federal \\ Fluminense, 24241-000 Niterói-RJ, Brazil
}

\begin{abstract}
Neste trabalho, verificou-se a aplicabilidade de uma metodologia computacional para se predizer a estrutura de compostos orgânicos com atividade biológica. Para isso, selecionaramse três floroglucinóis, e compararam-se suas conformações obtidas por modelagem molecular e por difração de raios X. Os resultados mostraram que as conformações obtidas por análise conformacional com o método AM1 seguidas de otimização de geometria utilizando o método DFT (B3LYP/6-31G(d,p)) estão em boa concordância com os dados obtidos experimentalmente por difração de raios $\mathrm{X}$, indicando que a metodologia empregada parece ser uma ótima ferramenta para predizer preferências conformacionais desta classe de compostos.
\end{abstract}

In this work we intend to verify the applicability of a computational methodology to predict structural features of organic compounds with biological activity. We selected three phloroglucinols and compared their calculated conformational data with their X-ray crystallographic structure. The results showed that conformations obtained by conformational analysis with the AM1 method followed by geometry optimization by using the DFT B3LYP/6-31 G(d,p) basis set are in very good agreement with X-ray data, indicating that the methodology employed here seems to be a very useful tool in order to predict the conformational preference for this class of compounds.

Keywords: conformational analysis, X-ray, molecular modeling, phloroglucinols

\section{Introduction}

Despite the development of organic synthesis, biotechnology and combinatorial chemistry, natural plants are still a great source of bioactive compounds. However, just $8 \%$ of the Brazilian flora has been already studied in the search for new bioactive substances. ${ }^{1,2}$

*e-mail: kzleal@uol.com.br
In the last decades several antibacterial compounds are being less effective in the treatment of infectious diseases due to multi-resistant bacteria. ${ }^{3}$ In this context medicinal plants are extremely important in the search of new molecules as therapeutic alternatives. The genus Hypericum seems to be a good choice for this problem. It is constituted by flavonoids, xantones and phloroglucinols with considerable pharmacological and biological effects. ${ }^{4,5}$ Phloroglucinol derivatives have been described in the literature as promising substances with 
high antibacterial activity. ${ }^{6-9}$ The Hypericum brasiliense presents three phloroglucinols in its constitution and they are active against bacteria. ${ }^{10,11}$ These phloroglucinols are the japonicin $\mathrm{A}$, the uliginosin $\mathrm{B}$ and the isouliginosin $\mathrm{B}$ (Figure 1).

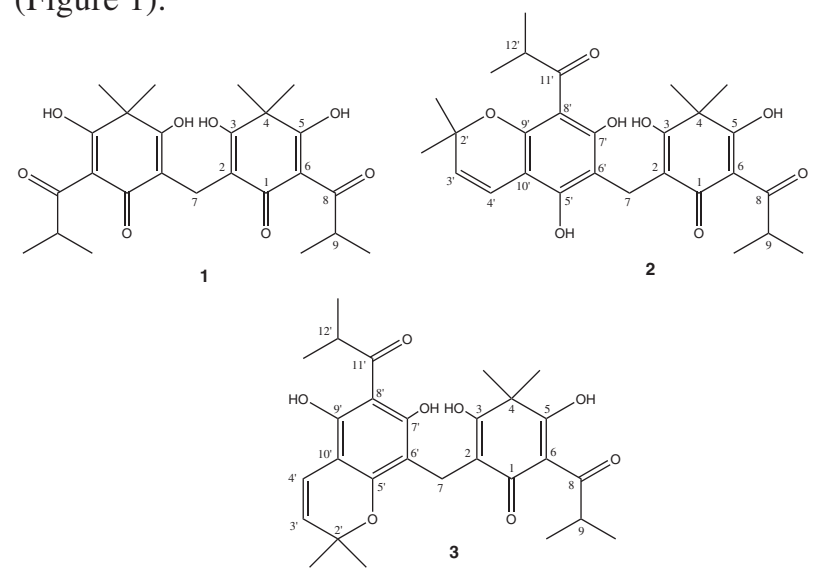

Figure 1. Phloroglucinols from Hypericum brasiliense: japonicin A (1), uliginosin B (2) and isouliginosin B (3) respectively.

The study of conformations of bioactive compounds is very important in the design of new drugs, since that conformational profile may have direct implications in their activity. When the conformational properties of a drug are known to play an important role in establishing its therapeutic value, any newly designed analogue should have similar conformational properties to enhance the probability that it will bind to the receptor target.

Nowadays computational chemistry is a very important tool in drug design. It can lead for example, to the determination of physical chemical properties and to conformational profiles of biomolecules. However, success in using conformational profiles in the study of biochemical interactions depends on how well computer simulation is able to reproduce the structural features of the species involved. ${ }^{12-17}$

In this work we intend to verify the applicability of a computational methodology to predict structural features of organic compounds with biological activity. We selected the phloroglucinols 1, 2 and $\mathbf{3}$ and compared their calculated conformational data with X-ray experimental results.

\section{Experimental}

The phloroglucinols used in this work were isolated from the Hypericum brasiliense. ${ }^{4,5}$ The structure of the compounds was established by comparing its NMR data $\left({ }^{1} \mathrm{H}\right.$ and ${ }^{13} \mathrm{C}$ spectra) with that from literature. ${ }^{4}$

Samples of japonicin A (1) and isouliginosin B (3) suitable for X-ray diffraction experiments were obtained by slow solvent evaporation at room temperature of solutions containing hexane. All attempts to obtain single crystals of uliginosin B (2) by slow solvent evaporation failed. Uliginosin B (2) data collection and data reduction was performed in a two fold-twinned sample crystallized after evaporation of acetonitrile solution.

$\mathrm{X}$-ray diffraction data collections were performed on a Oxford-Diffraction GEMINI diffractometer (LabCri) using graphite-Enhance Source MoK $\alpha$ radiation $(\lambda=0.71069 \AA)$ at $150(2) \mathrm{K}$. Data Integration and scaling of the reflections were performed with the Crysalis suite..$^{18}$ Final unit cell parameters were based on the fitting of all reflections positions. Empirical multiscan absorption corrections using equivalent reflections were performed with the program SCALE3 ABSPACK. ${ }^{19}$

The structures of all compounds were solved by direct methods using the SHELXS program..$^{20}$ For each compound, the positions of all atoms could be unambiguously assigned on consecutive difference Fourier maps. Refinements were performed using SHELXL ${ }^{20}$ based on $\mathrm{F}^{2}$ through full-matrix least square routine. For uliginosin B (2) the Crysalis suite help in finding the twin law to generate a reflection file for refinement with the HKLF5 option of the SHELXL program..$^{20}$ All hydrogen atoms were refined with anisotropic atomic displacement parameters. During the refinements neither solvent nor disordered groups were identified in the crystals.

Except for those in the hydroxyl groups, the hydrogen atoms in the compounds were added in the structure in idealized positions and further refined according to the riding model. ${ }^{21}$ Owing the high $\mathrm{X}$-ray diffraction data quality the coordinates of the $\mathrm{H}$ atoms of the hydroxyl groups in the japonicin A (1) and in isouliginosin B (3) were freely refined. The hydrogen atom from hydroxyl group bounded to $\mathrm{C} 3$ in the japonicin A (1) molecule as well as those in the hydroxyl groups in the uliginosin B (3) were identified trough the electron density peaks observed in the Fourier difference maps nearby the oxygen atoms. These peaks were associated to $\mathrm{H}$ atoms and their coordinates were refined according to the riding model..$^{21}$

The conformational search was done by the AM1 method using the Hyperchem 7.0 program. ${ }^{22}$ The geometry of the conformer of lowest energy was fully optimized using the B3LYP/6-31G(d,p) basis set on the Gaussian 98 package of molecular orbital programs. ${ }^{23}$

\section{Results and Discussion}

$\mathrm{X}$-ray results show that japonicin A (1) unit cell is composed by eight molecules, uliginosin B (2) by four molecules and isouliginosin B (3) by two molecules. In all compounds the molecules are bonded by a series of 
Table 1. Bond lengths $(\AA)$ calculated by the Gaussian 98 program and obtained by X-ray diffraction for the phloroglucinols studied

\begin{tabular}{|c|c|c|c|c|c|c|}
\hline & \multicolumn{2}{|c|}{ (1) } & \multicolumn{2}{|c|}{ (2) } & \multicolumn{2}{|c|}{ (3) } \\
\hline & Calculated & X-ray & Calculated & X-ray & Calculated & $\mathrm{X}$-ray \\
\hline $\mathrm{C} 1-\mathrm{C} 2$ & 1.45 & 1.45 & 1.46 & 1.45 & 1.46 & 1.46 \\
\hline $\mathrm{C} 2-\mathrm{C} 3$ & 1.36 & 1.35 & 1.36 & 1.34 & 1.36 & 1.35 \\
\hline $\mathrm{C} 2-\mathrm{C} 7$ & 1.51 & 1.51 & 1.52 & 1.52 & 1.52 & 1.52 \\
\hline C3-C4 & 1.51 & 1.50 & 1.51 & 1.50 & 1.51 & 1.51 \\
\hline C4-C5 & 1.51 & 1.52 & 1.51 & 1.50 & 1.51 & 1.51 \\
\hline C5-C6 & 1.40 & 1.40 & 1.40 & 1.40 & 1.40 & 1.39 \\
\hline $\mathrm{C} 6-\mathrm{C} 1$ & 1.47 & 1.47 & 1.47 & 1.47 & 1.47 & 1.47 \\
\hline C6-C8 & 1.47 & 1.44 & 1.47 & 1.45 & 1.47 & 1.46 \\
\hline C8-C9 & 1.52 & 1.50 & 1.52 & 1.51 & 1.52 & 1.50 \\
\hline $\mathrm{C} 3-\mathrm{OH}$ & 1.33 & 1.34 & 1.34 & 1.35 & 1.34 & 1.35 \\
\hline $\mathrm{C} 5-\mathrm{OH}$ & 1.31 & 1.30 & 1.31 & 1.30 & 1.31 & 1.30 \\
\hline $\mathrm{C} 1=\mathrm{O}$ & 1.26 & 1.25 & 1.25 & 1.25 & 1.25 & 1.25 \\
\hline $\mathrm{C} 8=\mathrm{O}$ & 1.26 & 1.26 & 1.26 & 1.27 & 1.26 & 1.26 \\
\hline C2'-C3' & - & - & 1.51 & 1.50 & 1.51 & 1.50 \\
\hline C3'-C4' & - & - & 1.34 & 1.33 & 1.34 & 1.33 \\
\hline C4'-C10' & - & - & 1.46 & 1.46 & 1.45 & 1.45 \\
\hline $\mathrm{C} 10^{\prime}-\mathrm{C} 5^{\prime}$ & - & - & 1.42 & 1.41 & 1.40 & 1.39 \\
\hline C5'-C6' & - & - & 1.41 & 1.39 & 1.40 & 1.39 \\
\hline C6'-C7' & - & - & 1.40 & 1.39 & 1.41 & 1.40 \\
\hline C6'-C7 & - & - & 1.52 & 1.51 & 1.52 & 1.52 \\
\hline C7'-C8' & - & - & 1.44 & 1.43 & 1.43 & 1.43 \\
\hline C8'-C9' & - & - & 1.43 & 1.42 & 1.44 & 1.42 \\
\hline C9'-C10' & - & - & 1.40 & 1.38 & 1.41 & 1.39 \\
\hline C8'-C11' & - & - & 1.46 & 1.47 & 1.47 & 1.47 \\
\hline $\mathrm{C} 11^{\prime}-\mathrm{C} 12^{\prime}$ & - & - & 1.53 & 1.51 & 1.53 & 1.52 \\
\hline $\mathrm{C} 2{ }^{\prime}-\mathrm{O}$ & - & - & 1.47 & 1.47 & 1.48 & 1.48 \\
\hline $\mathrm{C}^{\prime}-\mathrm{O}$ & - & - & - & - & 1.38 & 1.38 \\
\hline C9'-O & - & - & 1.36 & 1.36 & - & - \\
\hline $\mathrm{C}^{\prime}-\mathrm{OH}$ & - & - & 1.34 & 1.35 & - & - \\
\hline C7'-OH & - & - & 1.35 & 1.35 & 1.34 & 1.35 \\
\hline C9'-OH & - & - & - & - & 1.33 & 1.35 \\
\hline $\mathrm{C} 11^{\prime}=\mathrm{O}$ & - & - & 1.26 & 1.25 & 1.25 & 1.24 \\
\hline C7-H & 0.99 & 0.99 & 0.99 & 0.99 & 0.99 & 0.99 \\
\hline C9-H & 1.00 & 1.00 & 1.00 & 1.00 & 1.00 & 1.00 \\
\hline C4-C-H & 0.98 & 0.98 & 0.98 & 0.98 & 0.98 & 0.98 \\
\hline C9-C-H & 0.98 & 0.98 & 0.98 & 0.98 & 0.98 & 0.98 \\
\hline C3-O-H & 0.90 & 0.91 & 0.84 & 0.84 & 0.86 & 0.86 \\
\hline C5-O-H & 0.84 & 0.84 & 0.84 & 0.84 & 1.04 & 1.04 \\
\hline C3'-H & - & - & 0.95 & 0.95 & 0.95 & 0.95 \\
\hline $\mathrm{C} 4^{\prime}-\mathrm{H}$ & - & - & 0.95 & 0.95 & 0.95 & 0.95 \\
\hline C12'-H & - & - & 1.00 & 1.00 & 1.00 & 1.00 \\
\hline C2'-C-H & - & - & 0.98 & 0.98 & 0.98 & 0.98 \\
\hline C12'-C-H & - & - & 0.98 & 0.98 & 0.98 & 0.98 \\
\hline $\mathrm{C}^{\prime}-\mathrm{O}-\mathrm{H}$ & - & - & 0.84 & 0.84 & - & - \\
\hline C7'-O-H & - & - & 0.84 & 0.84 & 0.94 & 0.94 \\
\hline C9'-O-H & - & - & & - & 0.94 & 0.94 \\
\hline
\end{tabular}


Table 2. Interatomic distances $(\AA)$ for hydrogen bonds calculated by the Gaussian 98 program and obtained by X-ray diffraction for the phloroglucinols studied

\begin{tabular}{|c|c|c|c|c|c|c|}
\hline & \multicolumn{2}{|c|}{ (1) } & \multicolumn{2}{|c|}{ (2) } & \multicolumn{2}{|c|}{ (3) } \\
\hline & Calculated & X-ray & Calculated & X-ray & Calculated & $\mathrm{X}$-ray \\
\hline C1-O...HO-C3 & 1.62 & 1.73 & - & - & - & - \\
\hline C1-O...HO-C5 & 1.61 & 1.72 & - & - & - & - \\
\hline $\mathrm{C} 8=\mathrm{O} \ldots \mathrm{HO}-\mathrm{C} 3$ & 1.42 & 1.62 & - & - & - & - \\
\hline $\mathrm{C} 8=\mathrm{O} \ldots \mathrm{HO}-\mathrm{C} 5$ & 1.42 & 1.65 & - & - & - & - \\
\hline C1-O...HO-C5, & & & 1.64 & 1.80 & & \\
\hline C3-OH...OH-C7' & & & 1.71 & 1.88 & & \\
\hline $\mathrm{C} 8=\mathrm{O} \ldots \mathrm{HO}-\mathrm{C} 5$ & & & 1.42 & 1.65 & & \\
\hline $\mathrm{C} 11^{\prime}=\mathrm{O} . . . \mathrm{HO}-\mathrm{C} 7^{\prime}$ & & & 1.44 & 1.68 & & \\
\hline C1-O...HO-C7' & - & - & - & - & 1.63 & 1.69 \\
\hline C2'-O...HO-C3 & - & - & - & - & 1.75 & 1.90 \\
\hline $\mathrm{C} 8=\mathrm{O} \ldots \mathrm{HO}-\mathrm{C} 5$ & - & - & - & - & 1.41 & 1.41 \\
\hline $\mathrm{C} 11^{\prime}=\mathrm{O} . . . \mathrm{HO}-\mathrm{C} 9^{\prime}$ & - & - & - & - & 1.52 & 1.62 \\
\hline
\end{tabular}

weak hydrogen bonds and van deer Waals contacts. Crystal structure and refinement data for compounds (1), (2) and (3) are given as Supplementary Information. Calculated and experimental data are shown in Tables 1 and 2.

Structural parameters calculated by the DFT method on Tables 1 and 2 are in very good agreement with X-ray experimental data. Calculated bond lengths (Table 1) are within $0.03 \AA$ from the experimental ones. Calculations also reflect very well the hydrogen bond interactions (Table 2) which seem to be essential to keep the conformations of these phloroglucinols (Figure 2), since that these interactions correspond to hydrogen bonds of strong to moderate intensity according to Jeffrey, ${ }^{24}$ who defines that distances between $\mathrm{H} \cdots \mathrm{O}$ in the range of about 1.2-1.5 $\AA$ are related to strong interaction, distances of about $1.5-2.2 \AA$ correspond to a moderate interaction and distances of about 2.2-3.2 ̊̊ mean a weak one.
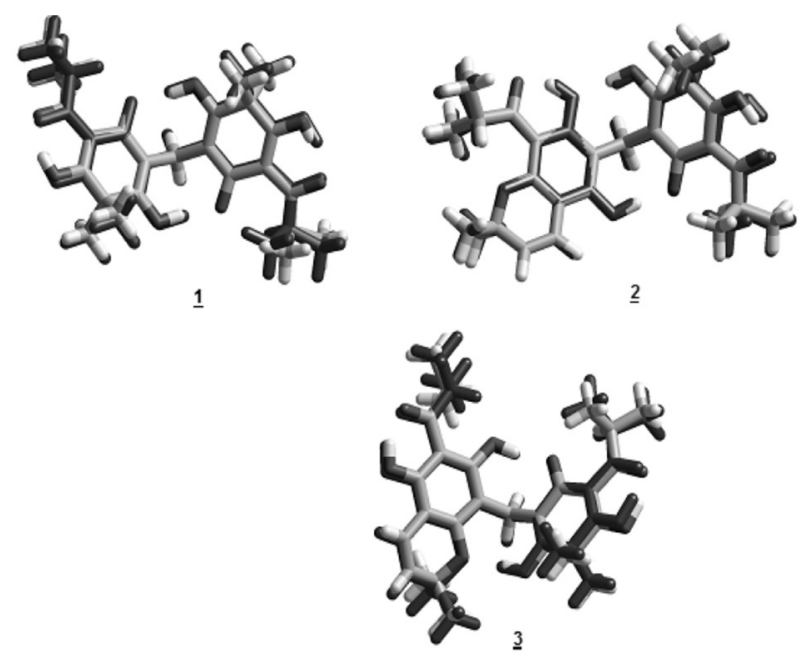

Figure 2. Conformations (obtained by molecular modeling - in gray - and by X-ray - in black) adopted by the phloroglucinols studied: japonicin A (1), uliginosin B (2) and isouliginosin B (3) respectively.
The conformations on Figure 2 reveals that this type of calculation should be extremely useful in predicting conformational preferences of similar structures since the geometries obtained experimentally by X-ray diffraction and theoretically by molecular modeling are very close $(\mathrm{RMS}=0.016 \AA$ for compound $(\mathbf{1})$ and $0.014 \AA$ for compounds (2) and (3)). It is important to notice that although the structure in solution is the most important one when we are studying biological activity, in the case focused here, changes in conformation will probably be minor due to the several hydrogen bonds.

\section{Conclusions}

Calculations using the DFT B3LYP/6-31 G(d,p) basis set showed very good agreement between structural parameters calculated at this level and X-ray data, indicating that the methodology employed here seems to be a very useful tool in order to predict the conformational preference for this class of compounds.

\section{Supplementary Information}

CCDC 737455-737457 contains the supplementary crystallographic data for the structures in this paper. These data can be obtained free of charge from the Director, CCDC, 12 Union Road, Cambridge, CB2 1EZ, UK (fax: +44-1223-336033; e-mail: deposit@ccdc.cam.ac.uk or http://www.ccdc.cam.ac.uk).

\section{Acknowledgements}

EBL held a graduate fellowship from FAPERJ and ALC from CNPq. 


\section{References}

1. Simões, C. M.; Farmacognosia da Planta ao Medicamento. $6^{\text {th }}$ ed., Editora da UFSC: Santa Catarina, 2007.

2. Morais, S. K. R.; Teixeira, A. F.; Torres, Z. E. D. S.; Nunomura, S. M.; Yamashiro-Kanashiro, E. H.; Lindoso, J. A. L.; Yoshida, M.; J. Braz. Chem. Soc. 2009, 20, 1110.

3. Von Eiff, C.; Peters, G.; Heilmann, C.; Lancet Infect. Dis. 2002, 2, 677.

4. França, H. S.; Kuster, R. M.; Riyo, P. N.; Oliveira, A. P.; Teixeira, L. A.; Rocha, L.; Quim. Nova 2009, 35, 1103.

5. França, H. S.; MSc Dissertation, Universidade Federal do Rio de Janeiro, Brazil, 2005.

6. Jayasuriya, H.; Clark, A. M.; Mcchfsney, J. D.; J. Nat. Prod. 1991, 54, 1314.

7. Li-Hong, H.; Ching-Wan, K.; Vittal, J. J.; Keng-Yeow, S.; Phytochemistry 2000, 53, 705.

8. Matsuhisa, M.; Shikishima, Y.; Takaishi, Y.; Honda, G.; Ito, M.; Takeda, Y.; Shibata, H.; Higuti, T.; Kodzhimatov, O. K.; Ashurmetov, O.; J. Nat. Prod. 2002, 65, 290.

9. Winkelmann, K.; Heilmann, J.; Zerbe, O.; Rali, T.; Sticher, O.; J. Nat. Prod. 2001, 64, 701.

10. Rocha, L.; Marston, A.; Potterat, O.; Kaplan, A. C.; StoeckliEvans, H.; Hostettmann, K.; Phytochemistry 1995, 40, 1447.

11. Rocha, L.; Marston, A.; Potterat, O.; Kaplan, A. C.; Hostettmann, K.; Phytochemistry 1996, 42, 185.

12. Leal, K. Z.; Seidl, P. R.; Yoneda, J. D.; dos Santos, C. V. B.; De Souza, M. C. B. V.; Ferreira, V. F.; J. Mol. Struct. 2005, 748, 137.

13. Braga, C. F.; Longo, R. L.; J. Braz. Chem. Soc. 2008, 19, 321.

14. Salum, L. B.; Dias, L. C.; Andricopulo, A. D.; J. Braz. Chem. Soc. 2009, 20, 693.
15. Zanatta, N.; Borchhardt, D. M.; Carpes, A. D.; Marchi, T. M. ; Andricopulo, A. D. ; Salum, L. B.; Schetinger, M. R. C.; Bonacorso, H. G.; Martins, M. A. P.; Flores, A. F. C.; J. Braz. Chem. Soc. 2008, 19, 1118.

16. França, T. C. C.; Rocha, M. R. M.; Reboredo, B. M.; Rennó, M. N.; Tinoco, L. W.; Villar, J. D. F.; J. Braz. Chem. Soc. 2008, 19, 64 .

17. Da Silva, J. B. P.; Ramos, M. N.; de Barros, B.; De Melo, S. J.; Falcão, E. P. S.; Catanho, M. T. J. A.; J. Braz. Chem. Soc. 2008, 19, 337.

18. Program CrysAlis-CCD and-RED, Oxford Diffraction Ltd., version 1.171.32.38.

19. SCALE3 ABSPACK scaling algorithm. CrysAlis RED, Oxford Diffraction Ltd., Version 1.171.32.38.

20. Sheldrick, G. M.; Schneider, T. R.; Methods Enzymol. 1997, 277, 319 .

21. Johnson, C. K.; Crystallographic Computing, Ahmed, F.R., ed.; Copenhagen, Munksgaard, 1970.

22. HyperChem(TM), Hypercube, Inc., $1115 \mathrm{NW} 4^{\text {th }}$ Street, Gainesville, Florida 32601, USA.

23. Frisch, M. J.; Trucks, G. W.; Schlegel, H. B.; Gill, P. M. W.; Johnson, B. G.; Robb, M. A.; Cheeseman, J. R.; Keith, T. G.; Peterson, G. A.; Montgomery, J. A.; Raghavachari, K.; AlLaham, M. A.; Zakrzewski, V. G.; Ortiz, J. V.; Foresman, J. B.; Cioslowski, J.; Stefanov, B. B.; Nanayakkara, A.; Challacombe, M.; Peng, C. Y.; Ayala, P. Y.; Chen, W.; Wong, N. W.; Andress, J. L.; Replogle, E. S.; Gomperts, R.; Martin, R. L.; Fox, D. L.; Binkley, J. S.; Defrees, D. J.; Baker, J.; Stewart, J. P.; HeadGordon, M.; Gonzalez, C.; Pople, J. A.; Gaussian 98. Gaussian Inc.: Pittsburg, PA, 1998.

24. Jeffrey, G. A.; An Introduction to Hydrogen Bonding, Oxford University Press: New York, 1997, ch. 2.

Received: November 27, 2009 Web Release Date: February 8, 2010 


\title{
Conformational Analysis of Phloroglucinols from Hypericum Brasiliense by using X-ray Diffraction and Molecular Modeling
}

\author{
Kátia Z. Leal, ${ }^{*, a, b}$ Julliane D. Yoneda ${ }^{c}$ Eric B. Lindgren ${ }^{a}$ Carlos B. Pinheiro, ${ }^{d}$ \\ Arthur L. Corrêa ${ }^{a}$ and Hildegardo S. França ${ }^{e}$
}

${ }^{a}$ Departamento de Físico-Química, Instituto de Química, Universidade Federal Fluminense, 00000-000 Niterói-RJ, Brazil

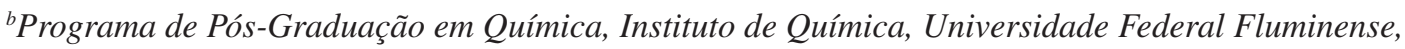
00000-000 Niterói-RJ, Brazil

cPólo Universitário de Volta Redonda, Universidade Federal Fluminense, 00000-000 Volta Redonda-RJ, Brazil

${ }^{d}$ Departamento de Física, Universidade Federal de Minas Gerais, 00000-000 Belo Horizonte-MG, Brazil.

${ }^{e}$ Departamento de Tecnologia Farmacêutica, Faculdade de Farmácia, Universidade Federal Fluminense, 00000-000 Niterói-RJ, Brazil

Table S1. Summary of the crystal structure data collection and refinement for japonicin A (1), uliginosin B (2) and isouliginosin B (3)

\begin{tabular}{|c|c|c|c|}
\hline Identification code & Japonicin A (1) & Uliginosin B (2) & Isouliginosin B (3) \\
\hline Empirical formula & $\mathrm{C}_{25} \mathrm{H}_{32} \mathrm{O}_{8}$ & $\mathrm{C}_{28} \mathrm{H}_{34} \mathrm{O}_{8}$ & $\mathrm{C}_{28} \mathrm{H}_{34} \mathrm{O}_{8}$ \\
\hline Formula weight & 460.51 & 498.55 & 498.55 \\
\hline Temperature & $150(2) \mathrm{K}$ & $150(2) \mathrm{K}$ & $150(2) \mathrm{K}$ \\
\hline Wavelength & $1.5418 \AA$ & $1.5418 \AA$ & $1.5418 \AA$ \\
\hline Crystal system & Monoclinic & Monoclinic & Triclinic \\
\hline Space group & $\mathrm{P} 21 / \mathrm{n}$ & $\mathrm{P} 21 / \mathrm{n}$ & $\mathrm{P}-1$ \\
\hline Unit cell dimensions & $\begin{array}{l}a=9.2461(4) \AA \\
b=17.8198(6) \AA \\
c=28.8240(10) \AA \\
\alpha=90^{\circ} . \\
\beta=90^{\circ} . \\
\gamma=90^{\circ}\end{array}$ & $\begin{array}{l}a=10.4085(4) \AA \\
b=9.1035(3) \AA \\
c=27.5920(10) \AA \\
\alpha=90.000(3)^{\circ} . \\
\beta=99.070(3)^{\circ} . \\
\gamma=90.000(3)^{\circ} .\end{array}$ & $\begin{array}{l}a=10.4944(4) \AA \\
b=10.7537(3) \AA \\
c=12.3616(4) \AA \\
\alpha=103.610(3)^{\circ} . \\
\beta=110.336(3)^{\circ} . \\
\gamma=93.969(3)^{\circ}\end{array}$ \\
\hline Volume & $4749.1(3) \AA^{3}$ & $2581.76(16) \AA^{3}$ & $1253.80(8) \AA^{3}$ \\
\hline $\mathrm{Z}$ & 8 & 4 & 2 \\
\hline Density (calculated) & $1.288 \mathrm{Mg} / \mathrm{m}^{3}$ & $1.283 \mathrm{Mg} / \mathrm{m}^{3}$ & $1.321 \mathrm{Mg} / \mathrm{m}^{3}$ \\
\hline Absorption coefficient & $0.792 \mathrm{~mm}^{-1}$ & $0.77 \mathrm{~mm}^{-1}$ & $0.793 \mathrm{~mm}^{-1}$ \\
\hline $\mathrm{F}(000)$ & 1968 & 1064 & 532 \\
\hline Crystal size & $0.39 \times 0.11 \times 0.06 \mathrm{~mm}^{3}$ & $0.49 \times 0.14 \times 0.03 \mathrm{~mm}^{3}$ & $0.38 \times 0.22 \times 0.09 \mathrm{~mm}^{3}$ \\
\hline Theta range for data collection & 3.07 to $62.48^{\circ}$. & 3.24 to $62.68^{\circ}$ & 3.97 to $62.89^{\circ}$ \\
\hline Index ranges & $\begin{array}{l}-10<=\mathrm{h}<=10 \\
-20<=\mathrm{k}<=20 \\
-33<=1<=33\end{array}$ & $\begin{array}{l}-11<=\mathrm{h}<=11, \\
-10<=\mathrm{k}<=10, \\
-31<=\mathrm{l}<=31\end{array}$ & $\begin{array}{l}-12<=\mathrm{h}<=11, \\
-12<=\mathrm{k}<=12, \\
-14<=\mathrm{l}<=14\end{array}$ \\
\hline Reflections collected & 39758 & 6833 & 32807 \\
\hline
\end{tabular}

*e-mail: kzleal@uol.com.br 
Table S1. continuation

\begin{tabular}{|c|c|c|c|}
\hline Identification code & Japonicin A (1) & Uliginosin B (2) & Isouliginosin B (3) \\
\hline Independent reflections & $3776[\mathrm{R}(\mathrm{int})=0.0330]$ & $6973[\mathrm{R}(\mathrm{int})=0.0000]$ & $3958[\mathrm{R}(\mathrm{int})=0.0331]$ \\
\hline Completeness to theta $=62.89^{\circ}$ & $99.8 \%$ & $98.5 \%$ & $98.0 \%$ \\
\hline Absorption correction & Analytical & Analytical & Semi-empirical from equivalents \\
\hline Max. and min. transmission & 0.962 and 0.829 & 0.937 and 0.552 & 0.9338 and 0.7521 \\
\hline Refinement method & Full-matrix least-squares on $\mathrm{F}^{2}$ & Full-matrix least-squares on $\mathrm{F}^{2}$ & Full-matrix least-squares on $\mathrm{F}^{2}$ \\
\hline Data / restraints / parameters & $3776 / 0 / 309$ & $6973 / 12$ / 327 & $3958 / 0 / 342$ \\
\hline Goodness-of-fit on $\mathrm{F}^{2}$ & 1.060 & 1.059 & 1.042 \\
\hline Final $\mathrm{R}$ indices $[\mathrm{I}>2 \operatorname{sigma}(\mathrm{I})]$ & $\begin{array}{l}\mathrm{R} 1=0.0510 \\
\mathrm{wR} 2=0.1412\end{array}$ & $\begin{array}{l}R 1=0.0599 \\
w R 2=0.1644\end{array}$ & $\begin{array}{l}\mathrm{R} 1=0.0381, \\
\mathrm{wR} 2=0.1058\end{array}$ \\
\hline $\mathrm{R}$ indices (all data) & $\begin{array}{l}\mathrm{R} 1=0.0627, \\
\mathrm{wR} 2=0.1490\end{array}$ & $\begin{array}{l}\mathrm{R} 1=0.0902, \\
\mathrm{wR} 2=0.1767\end{array}$ & $\begin{array}{l}\mathrm{R} 1=0.0463 \\
\mathrm{wR} 2=0.1131\end{array}$ \\
\hline Extinction coefficient & $0.00008(4)$ & $0.00009(7)$ & $0.0009(3)$ \\
\hline Largest diff. peak and hole & 0.590 and -0.247 e. $\AA^{-3}$ & 0.269 and -0.266 e. $\AA^{-3}$ & 0.307 and -0.211 e. $\AA^{-3}$ \\
\hline
\end{tabular}

